\title{
Kinematical data on early-type galaxies. II. ${ }^{\star, \star \star}$
}

\author{
F. Simien and Ph. Prugniel \\ CRAL-Observatoire de Lyon (CNRS: UMR 142), F-69561 St-Genis-Laval Cedex, France
}

Received November 6, 1996; accepted February 14, 1997

\begin{abstract}
We present new kinematical data for a sample of 38 early-type galaxies. Rotation curves and velocitydispersion profiles are determined for 32 objects, while the central velocity dispersions are given for the whole sample. This is our second paper in a series devoted to the presentation of kinematical data on elliptical and S0 galaxies, derived from long-slit absorption spectroscopy.
\end{abstract}

Key words: galaxies: elliptical \& lenticular, cD galaxies: kinematics and dynamics - galaxies: fundamental parameters

\section{Introduction}

We have recently begun to present kinematical measurements from absorption spectroscopy on early-type galaxies (Simien \& Prugniel 1997, hereafter Paper I); these data are intended to contribute to the study of several structural and evolutionary issues. As part of our continued effort to get reliable velocity dispersions and rotation curves on a statistically significant sample of objects, we presently report on new observations on a second, and longer, list of targets.

This work follows closely the technique already described in full detail in Paper I, for both observation and reduction, and only a minimum of explanations will be given here.

\section{Sample and observations}

Our present sample consists of 38 early-type galaxies, with ellipticals and lenticulars in roughly equal proportions. This set adds up to the 21-object sample of Paper I;

Send offprint requests to: F. Simien (simien@obs.univ-lyon1.fr)

* Based on observations collected at the Observatoire de Haute-Provence.

** Tables 2 and 4 are presented in electronic form only; Tables 1 through 4 are available at the CDS via anonymous $\mathrm{ftp}$ to cdsarc.u-strasbg.fr (130.79.128.5) or via http://cdsweb.ustrasbg.fr/Abstract.html there are four objects in common (NGC 2329, NGC 2332, NGC 4434 and UGC 3696), whose new measurements have been included in the present work for comparison purposes. Relevant catalog elements are presented in Table 1. The Es have ellipticities corresponding to classes between $\simeq \mathrm{E} 0$ and $\simeq \mathrm{E} 4$, and the S0s are moderately to highly flattened. The distances are in the range of $\simeq 15$ to $\simeq 100 \mathrm{Mpc}\left(\right.$ for $\left.H_{0}=75 \mathrm{~km} \mathrm{~s}^{-1} \mathrm{Mpc}^{-1}\right)$. The absolute magnitudes are intermediate $\left(-21.8<M_{B}<-17.3\right)$.

The observations have been secured at the $1.93-\mathrm{m}$ telescope of the Observatoire de Haute-Provence, equipped with the CARELEC long-slit spectrograph. In March and June 1995, two observing runs totalling 12 nights collected spectra on the major axis of the galaxies.

The atmospheric conditions were variable, with a seeing disk between $2^{\prime \prime}$ and $3.5^{\prime \prime}$ (FWHM) for most objects, but up to $\simeq 5^{\prime \prime}$ for three of them. The log of the observations is given in Table 2, which is proposed in electronic form only.

\section{Data reduction}

As in Paper I, standard pre-processing was applied to the raw data, up to the rebinning in wavelength. The galaxy centers $(r=0)$ were determined by a gaussian fitting to a limited range $\left(\simeq 12^{\prime \prime}\right)$ around the brightest line. In the outer regions, cosmic-ray hits were removed with a median filter, and adjacent lines were combined with a variable weighting function (a gaussian continuously wider faintward). A Fourier-Fitting technique determined the central velocity dispersion $\sigma_{0}$ and, when possible, the radial profile $\sigma(r)$ of the dispersion, together with the projected rotation curve $V(r)$ along the major axis. A two-pass mode (described in Paper I) allowed to remove cosmics on the inner lines, where the spatial resolution must be preserved. We adopted as the systemic velocity the value measured at $r=0$. Whenever possible, we have measured the maximum rotation velocity $V_{\max }$, as the mean of representative values on the opposite semi-axes. 
Table 1. Catalog elements

\begin{tabular}{|c|c|c|c|c|c|c|c|c|c|c|c|c|}
\hline Object & Type & $\alpha_{1950}$ & $\delta_{1950}$ & $v_{\text {hel }}$ & $B_{\mathrm{T}}$ & $-M_{B}$ & $r_{\mathrm{e}}$ & $\epsilon$ & $\mathrm{PA}$ & ref. & $\sigma_{0}$ & $V_{\max }$ \\
\hline$(1)$ & $(2)$ & (3) & $(4)$ & $(5)$ & $(6)$ & (7) & $(8)$ & $(9)$ & $(10)$ & $(11)$ & $(12)$ & $(13)$ \\
\hline NGC 1521 & -5 & 040608.0 & -211100 & 4183 & 12.32 & 21.22 & 34.7 & 0.22 & 19 & 1 & $236 \pm 36$ & $\ldots \pm \ldots$ \\
\hline NGC 1653 & -4 & 044316.5 & -022853 & 4345 & 12.60 & 20.92 & 25.4 & 0.08 & 118 & 1 & $253 \pm 55$ & $\ldots \pm \ldots$ \\
\hline NGC 2329 & -3 & 070521.7 & 484148 & 5726 & 12.97 & 21.29 & 21.5 & 0.14 & 175 & 3 & $244 \pm 19$ & $99 \pm 05$ \\
\hline NGC 2332 & -2 & 070543.3 & 501548 & 5809 & 13.52 & 20.74 & $\ldots$ & 0.32 & 60 & 3 & $\ldots \pm \ldots$ & $\ldots \pm \ldots$ \\
\hline NGC 2563 & -2 & 081740.7 & 211340 & 4583 & 12.94 & 20.93 & 21.1 & 0.15 & 80 & 3 & $260 \pm 26$ & $\ldots \pm \ldots$ \\
\hline NGC 2695 & -2 & 085155.8 & -025236 & 1825 & 12.81 & 19.38 & 14.9 & 0.28 & 175 & 3 & $192 \pm 19$ & $\ldots \pm \ldots$ \\
\hline NGC 2986 & -5 & 094157.0 & -210254 & 2313 & 11.65 & 20.76 & 34.5 & 0.12 & 43 & 1 & $253 \pm 21$ & $\ldots \pm \ldots$ \\
\hline NGC 3853 & -5 & 114153.3 & 165010 & 3349 & 13.31 & 20.03 & 14.1 & 0.33 & 140 & 1 & $170 \pm 19$ & $\ldots \pm \ldots$ \\
\hline NGC 3862 & -5 & 114229.1 & 195305 & 6474 & 13.23 & 21.34 & 31.2 & 0.03 & 84 & 1 & $259 \pm 18$ & $\ldots \pm \ldots$ \\
\hline NGC 3921 & 0 & 114828.0 & 552120 & 5839 & 13.05 & 21.26 & 18.1 & 0.38 & 20 & 3 & $200 \pm 53$ & $\ldots \pm \ldots$ \\
\hline NGC 4270 & -2 & 121715.4 & 054431 & 2484 & 13.05 & 19.44 & 13.4 & 0.53 & 110 & 3 & $196 \pm 22$ & $\ldots \pm \ldots$ \\
\hline NGC 4283 & -5 & 121750.3 & 293518 & 727 & 12.88 & 18.17 & 13.1 & 0.10 & 56 & 1 & $103 \pm 10$ & $\ldots \pm \ldots$ \\
\hline NGC 4318 & -5 & 122010.6 & 082833 & 1383 & 14.11 & 16.90 & 7.2 & 0.33 & 67 & 1 & $101 \pm 11$ & $\ldots \pm \ldots$ \\
\hline NGC 4339 & -5 & 122101.3 & 062132 & 1282 & 12.26 & 18.75 & 32.4 & 0.16 & 134 & 1 & $119 \pm 18$ & $\ldots \pm \ldots$ \\
\hline NGC 4340 & -1 & 122103.7 & 170006 & 911 & 11.80 & 19.21 & 50.4 & 0.29 & 85 & 2 & $115 \pm 11$ & $\ldots \pm \ldots$ \\
\hline NGC 4429 & -1 & 122454.1 & 112305 & 1130 & 10.63 & 20.38 & 68.6 & 0.54 & 93 & 2 & $181 \pm 29$ & $\ldots \pm \ldots$ \\
\hline NGC 4434 & -5 & 122504.2 & 082553 & 1064 & 13.03 & 17.98 & 12.9 & 0.09 & 33 & 1 & $118 \pm 15$ & $\ldots \pm \ldots$ \\
\hline NGC 4435 & -2 & 122508.6 & 132123 & 786 & 11.56 & 19.45 & 23.9 & 0.38 & 13 & 2 & $165 \pm 16$ & $\ldots \pm \ldots$ \\
\hline NGC 4442 & -2 & 122531.3 & 100453 & 535 & 11.30 & 19.71 & 22.5 & 0.60 & 87 & 3 & $216 \pm 35$ & $\ldots \pm \ldots$ \\
\hline NGC 4461 & -1 & 122631.1 & 132743 & 1924 & 11.95 & 19.06 & 22.4 & 0.36 & 10 & 1 & $161 \pm 15$ & $\ldots \pm \ldots$ \\
\hline NGC 4464 & -1 & 122648.1 & 082605 & 1236 & 13.52 & 17.49 & 6.6 & 0.19 & 1 & 1 & $120 \pm 09$ & $\ldots \pm \ldots$ \\
\hline NGC 4468 & -3 & 122659.6 & 141933 & 895 & 13.70 & 17.31 & 15.9 & 0.28 & 72 & 2 & $75 \pm 91$ & $\ldots \pm \ldots$ \\
\hline NGC 4479 & -2 & 122746.8 & 135115 & 822 & 13.29 & 17.72 & 26.7 & 0.14 & 14 & 2 & $\ldots \pm \ldots$ & $\ldots \pm \ldots$ \\
\hline NGC 4489 & -5 & 122821.1 & 170205 & 934 & 12.68 & 18.33 & 33.0 & 0.11 & 160 & 1 & $63 \pm 15$ & $\ldots \pm \ldots$ \\
\hline NGC 4552 & -5 & 123308.4 & 124956 & 263 & 10.43 & 20.58 & 56.0 & 0.08 & 115 & 1 & $263 \pm 15$ & $1 \pm 05$ \\
\hline NGC 4638 & -3 & 124016.4 & 114300 & 1114 & 12.16 & 18.85 & 11.9 & 0.50 & 125 & 2 & $129 \pm 09$ & $150 \pm 10$ \\
\hline NGC 4874 & -4 & 125711.1 & 281353 & 7127 & 12.25 & 22.48 & 63.4 & 0.15 & 37 & 1 & $275 \pm 25$ & $7 \pm 10$ \\
\hline NGC 4886 & -5 & 125740.0 & 281532 & 6319 & 15.00 & 19.73 & 6.5 & 0.10 & 89 & 1 & $154 \pm 11$ & $\ldots \pm \ldots$ \\
\hline NGC 5353 & -2 & 135119.8 & 403147 & 2306 & 11.84 & 20.86 & 21.9 & 0.29 & 145 & 3 & $294 \pm 15$ & $\ldots \pm \ldots$ \\
\hline NGC 5424 & -2 & 140028.2 & 093938 & 5940 & 13.78 & 20.66 & 15.9 & 0.24 & 111 & 1 & $189 \pm 42$ & $\ldots \pm \ldots$ \\
\hline NGC 5490 & -5 & 140734.9 & 174653 & 5008 & 12.88 & 21.29 & 21.8 & 0.19 & 94 & 1 & $272 \pm 35$ & $\ldots \pm \ldots$ \\
\hline NGC 5557 & -5 & 141620.4 & 364325 & 3227 & 11.85 & 21.33 & 30.1 & 0.20 & 99 & 1 & $259 \pm 26$ & $\ldots \pm \ldots$ \\
\hline NGC 5854 & -1 & 150516.7 & 024537 & 1675 & 12.60 & 19.20 & 12.9 & 0.46 & 63 & 1 & $145 \pm 16$ & $\ldots \pm \ldots$ \\
\hline NGC 5864 & -2 & 150702.7 & 031433 & 1838 & 12.50 & 19.30 & 21.4 & 0.58 & 55 & 1 & $166 \pm 23$ & $\ldots \pm \ldots$ \\
\hline NGC 5966 & -5 & 153404.4 & 395603 & 4525 & 12.93 & 20.88 & 28.5 & 0.38 & 84 & 1 & $172 \pm 24$ & $\ldots \pm \ldots$ \\
\hline NGC 6146 & -5 & 162329.5 & 410024 & 8860 & 13.38 & 21.78 & 13.7 & 0.23 & 75 & 1 & $251 \pm 32$ & $\ldots \pm \ldots$ \\
\hline UGC 3696 & -5 & 070536.9 & 484258 & 5892 & 13.60 & 20.66 & 11.2 & 0.28 & 77 & 3 & $260 \pm 26$ & $\ldots \pm \ldots$ \\
\hline UGC 3792 & 0 & 071525.7 & 512304 & 5973 & 13.43 & 20.83 & $\cdots$ & 0.28 & 65 & 3 & $\ldots \pm \ldots$ & $\ldots \pm \ldots$ \\
\hline
\end{tabular}

\section{Notes:}

Col. (2): morphological type (from the LEDA database);

Cols. (3), (4): coordinates;

Col. (5): $v_{\text {hel }}$, heliocentric radial velocity, in $\mathrm{km} \mathrm{s}^{-1}$ (from $L E D A$ );

Col. (6): $B_{\mathrm{T}}$, integrated blue magnitude, corrected for Galactic extinction and $k$ term (as calculated by Prugniel \& Simien 1997 , hereafter PS97; except for NGC 2332 and UGC 3792, for which $B_{\mathrm{T}}$ is from $L E D A$ );

Col. (7): $-M_{B}$, absolute $B$ magnitude (for a distance modulus from PS97, corresponding to $H_{0}=75 \mathrm{~km} \mathrm{~s}^{-1} \mathrm{Mpc}^{-1}$ );

Col. (8): $r_{\mathrm{e}}$, effective radius (from PS97), in arcsec;

Col. (9): $\epsilon$, ellipticity;

Col. (10): PA, position angle of major axis, in degrees (North through East);

Col. (11): reference for $\epsilon$ and PA, $1=$ Djorgovski (1985), $2=$ Michard (1985), $3=L E D A$;

Col. (12): $\sigma_{0}$, central velocity dispersion available in the literature, from an updated version of the compilation in PS96 (excluding all previous measurements by ourselves), in $\mathrm{km} \mathrm{s}^{-1}$;

Col. (13): $V_{\max }$, maximum rotation velocity available in the literature, from PS96 (same remarks as for $\sigma_{0}$ ), in $\mathrm{km} \mathrm{s}^{-1}$. 

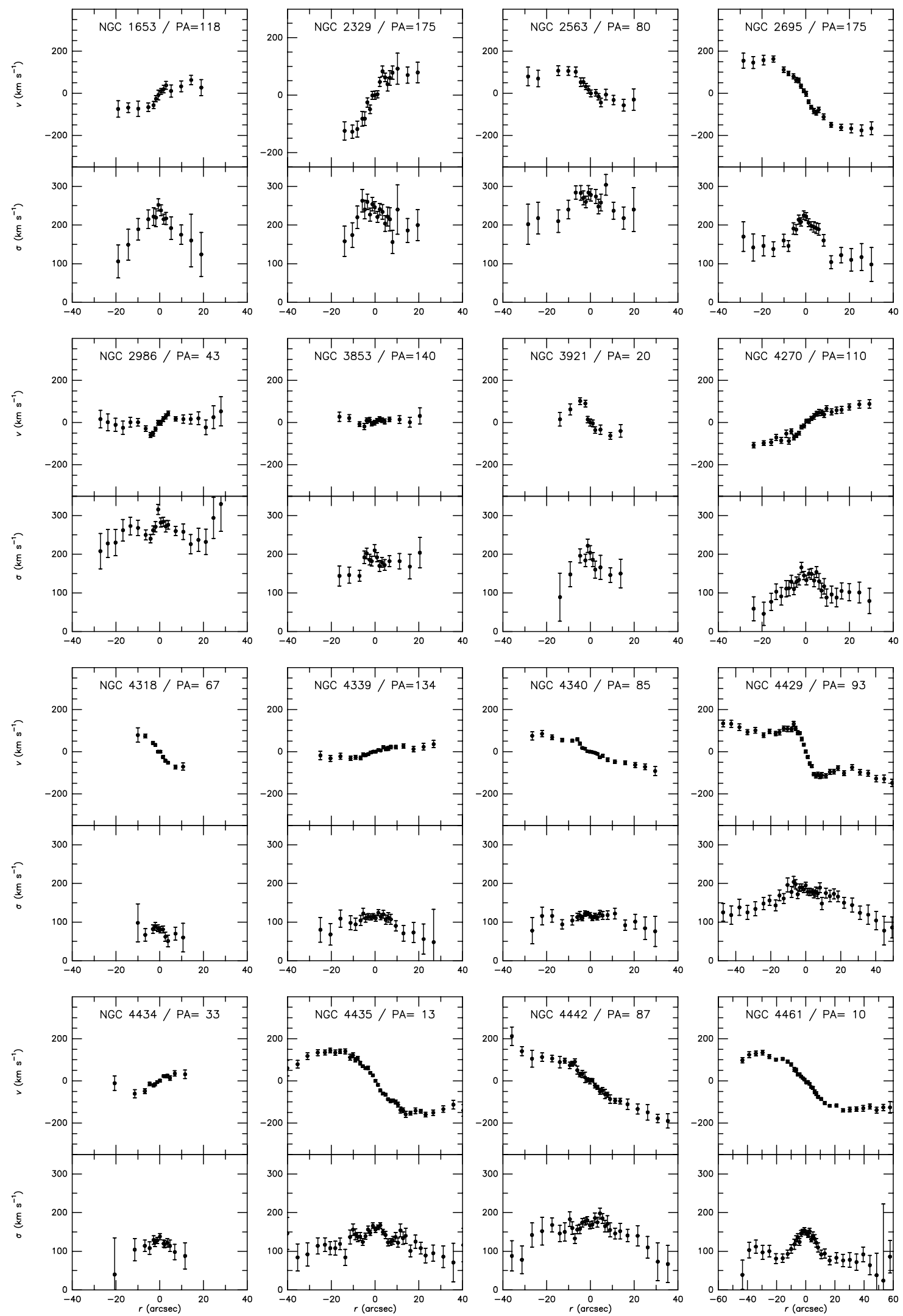

Fig. 1. Profiles of rotation velocities and velocity dispersions 

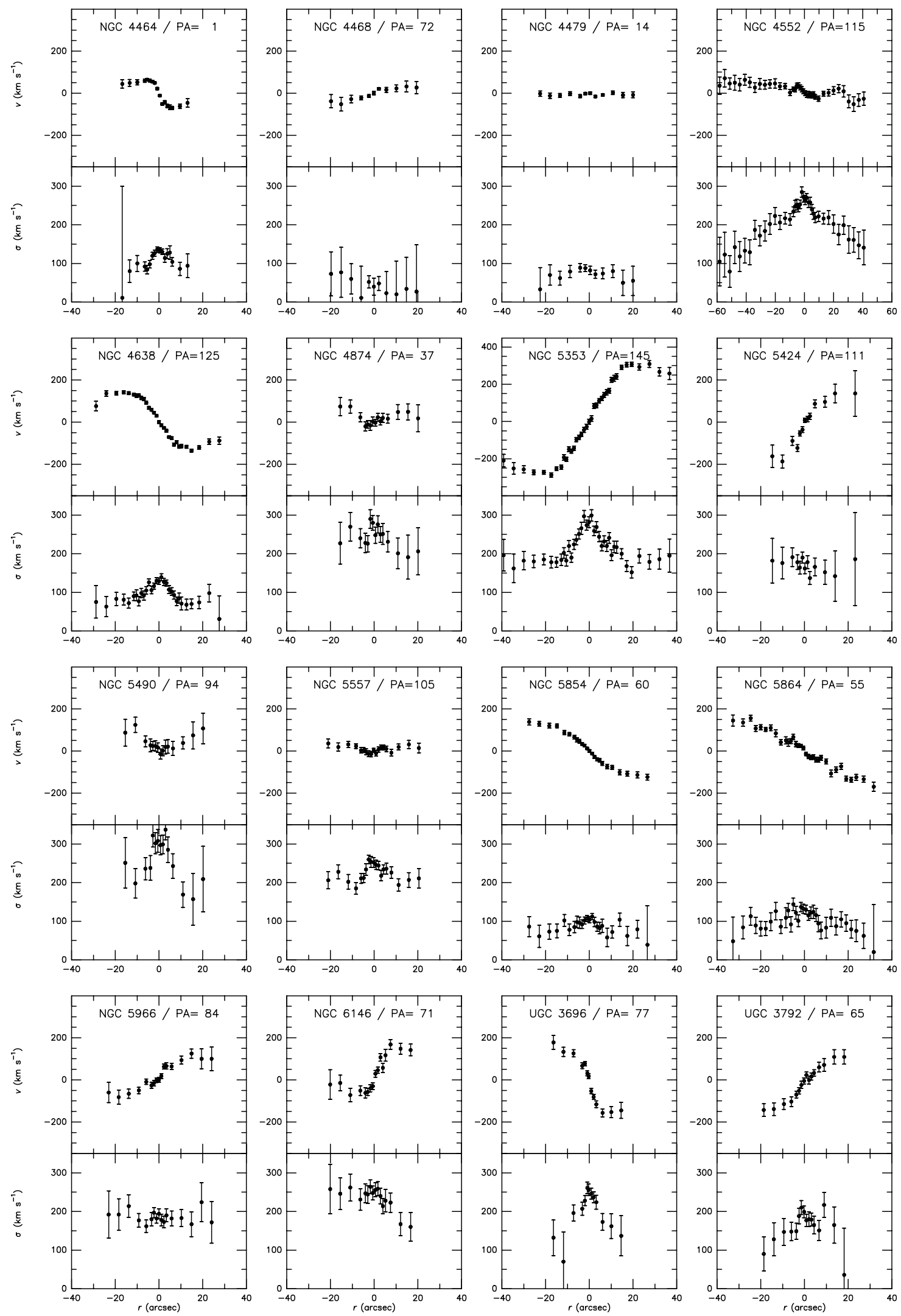

Fig. 1. continued 


\section{Presentation of the results}

Determinations of the heliocentric radial velocity $v_{\text {hel }}$, of $\sigma_{0}$, and of $V_{\max }$ (together with the corresponding radius $\left.r_{\max }\right)$ are listed in Table 3. The $V(r)$ and $\sigma(r)$ profiles are presented in Fig. 1, and also in Table 4, which is proposed in electronic form only. Tables 1 through 4 are available from the CDS.

Our results are summarized as follows:

- We have determined the central velocity dispersion $\sigma_{0}$ for $38 \mathrm{E}$ and $\mathrm{S} 0$ galaxies; in the literature (excluding Paper I and all previous measurements by ourselves), $\sigma_{0}$ was still unavailable for three of these objects (NGC 2332, NGC 4479 and UGC 3792).

- For 32 galaxies in our sample, we have been able to measure the $\sigma(r)$ and $V(r)$ profiles along the major axis. For 22 of these, the profiles extend beyond the effective radius. Whenever possible (for 30 galaxies), we have determined the maximum rotation velocity $V_{\max }$ : this parameter was still unavailable for 27 of these objects.

- In about half a dozen galaxies, there is an apparent asymmetry in the rotation curve; in the particular case of NGC 3921, there is also a clear asymmetry of the photometric profile outside $r \gtrsim 5^{\prime \prime}$. For three other galaxies (NGC 5490, and, marginally, NGC 4874 and NGC 5557), the rotation looks aberrant in the outer regions, being apparently in opposite sense for $r<0$ and $r>0$; deeper spectra would be valuable to settle the question. We note cases of asymmetry in the inner dispersion profiles, and also secondary peaks of $\sigma(r)$ (e.g., NGC 4270, NGC 4435, and NGC 5353), which could be artefacts caused by a strongly non-gaussian LOSVD (line-of-sight velocity distribution).

Acknowledgements. We are endebted to the telescope operators at the Observatoire de Haute-Provence for their continuous support. We are pleased to thank A. Pharasyn for his help during one of the observing runs, and the referee, G. Busarello, for his valuable comments. We have made use of the LyonMeudon Extragalactic Database supplied by the $L E D A$ team.

\section{References}

Djorgovski S., 1985, PhD thesis. Univ. of California, Berkeley Michard R., 1985, A\&AS 59, 205

Prugniel Ph., Simien F., 1996, A\&A 309, 749 (PS96)

Prugniel Ph., Simien F., 1997, A\&A 321, 111 (PS97)

Simien F., Prugniel Ph., 1997, A\&AS 122, 521 (Paper I)
Table 3. Kinematical results

\begin{tabular}{lrrrr}
\hline Object & $v_{\text {hel }}$ & $\sigma_{0}$ & $V_{\max }$ & $r_{\max }$ \\
\hline$(1)$ & $(2)$ & $(3)$ & $(4)$ & $(5)$ \\
\hline NGC 1521 & $4216 \pm 26$ & $233 \pm 24$ & $\ldots \pm \ldots$ & $\ldots$ \\
NGC 1653 & $4331 \pm 14$ & $245 \pm 14$ & $66 \pm 23$ & 15 \\
NGC 2329 & $5794 \pm 16$ & $246 \pm 15$ & $99 \pm 15$ & 20 \\
NGC 2332 & $5770 \pm 23$ & $237 \pm 20$ & $\ldots \pm \ldots$ & $\ldots$ \\
NGC 2563 & $4480 \pm 18$ & $278 \pm 16$ & $109 \pm 18$ & 15 \\
NGC 2695 & $1836 \pm 13$ & $222 \pm 10$ & $169 \pm 16$ & 20 \\
NGC 2986 & $2329 \pm 18$ & $282 \pm 12$ & $52 \pm 08$ & 08 \\
NGC 3853 & $3316 \pm 13$ & $207 \pm 15$ & $<25 \pm \ldots$ & 10 \\
NGC 3862 & $6429 \pm 19$ & $254 \pm 20$ & $\ldots \pm \ldots$ & $\ldots$ \\
NGC 3921 & $5839 \pm 19$ & $186 \pm 18$ & $80 ? \pm 30$ & 08 \\
NGC 4270 & $2357 \pm 11$ & $139 \pm 12$ & $97 \pm 11$ & 24 \\
NGC 4283 & $1045 \pm 14$ & $140 \pm 15$ & $\ldots \pm \ldots$ & $\ldots$ \\
NGC 4318 & $1231 \pm 09$ & $81 \pm 09$ & $75 \pm 20$ & 10 \\
NGC 4339 & $1289 \pm 09$ & $111 \pm 08$ & $28 \pm 11$ & 21 \\
NGC 4340 & $950 \pm 09$ & $118 \pm 07$ & $80 \pm 11$ & 22 \\
NGC 4429 & $1106 \pm 10$ & $195 \pm 08$ & $130 \pm 12$ & 43 \\
NGC 4434 & $1071 \pm 10$ & $137 \pm 08$ & $43 \pm 20$ & 20 \\
NGC 4435 & $801 \pm 10$ & $156 \pm 07$ & $152 \pm 08$ & 21 \\
NGC 4442 & $547 \pm 11$ & $170 \pm 09$ & $113 \pm 16$ & 20 \\
NGC 4461 & $1931 \pm 09$ & $150 \pm 06$ & $137 \pm 08$ & 30 \\
NGC 4464 & $1287 \pm 00$ & $135 \pm 07$ & $66 \pm 05$ & 05 \\
NGC 4468 & $909 \pm 11$ & $40 \pm 22$ & $32 \pm 22$ & 20 \\
NGC 4479 & $876 \pm 10$ & $82 \pm 11$ & $<10 \pm \ldots$ & $\ldots$ \\
NGC 4489 & $971 \pm 10$ & $63 \pm 13$ & $\ldots \pm \ldots$ & $\ldots$ \\
NGC 4552 & $364 \pm 14$ & $268 \pm 12$ & $17 \pm 10$ & 08 \\
NGC 4638 & $1164 \pm 10$ & $129 \pm 08$ & $139 \pm 07$ & 17 \\
NGC 4874 & $7129 \pm 24$ & $267 \pm 20$ & $\ldots \pm \ldots$ & $\ldots$ \\
NGC 4886 & $6317 \pm 14$ & $158 \pm 14$ & $\ldots \pm \ldots$ & $\ldots$ \\
NGC 5353 & $2186 \pm 16$ & $284 \pm 14$ & $298 \pm 09$ & 18 \\
NGC 5424 & $5951 \pm 15$ & $168 \pm 14$ & $150 \pm 34$ & 15 \\
NGC 5490 & $4855 \pm 24$ & $303 \pm 29$ & $\ldots \pm \ldots$ & $\ldots$ \\
NGC 5557 & $3213 \pm 13$ & $251 \pm 11$ & $<16 \pm \ldots$ & $\ldots$ \\
NGC 5854 & $1663 \pm 10$ & $104 \pm 08$ & $128 \pm 09$ & 25 \\
NGC 5864 & $1885 \pm 12$ & $131 \pm 12$ & $135 \pm 15$ & 28 \\
NGC 5966 & $4474 \pm 14$ & $194 \pm 13$ & $93 \pm 30$ & 18 \\
NGC 6146 & $8689 \pm 18$ & $252 \pm 18$ & $110 \pm 21$ & 12 \\
UGC 3696 & $6231 \pm 14$ & $253 \pm 15$ & $145 \pm 19$ & 11 \\
UGC 3792 & $6178 \pm 16$ & $195 \pm 15$ & $126 \pm 23$ & 18 \\
\hline & & & &
\end{tabular}

Notes:

Col. (2): $v_{\text {hel }}$, heliocentric radial velocity, in $\mathrm{km} \mathrm{s}^{-1}$; Col. (3): $\sigma_{0}$, central velocity dispersion, in $\mathrm{km} \mathrm{s}^{-1}$;

Col. (4): $V_{\max }$, maximum rotation velocity, in $\mathrm{km} \mathrm{s}^{-1}$; Col. (5): $r_{\max }$, the radius at which $V_{\max }$ has been measured, in arcsec. 